\title{
VORTICITY TRANSPORT ANALYSIS IN MAGNETIC VISCOELASTIC FLUID
}

\author{
Pardeep Kumar, Hari Mohan \\ Department of Mathematics, ICDEOL, Himachal Pradesh University, Shimla, India \\ e-mail: pkdureja@gmail.com \\ GAMAL HOSHOUDY \\ Department of Applied Mathematics, Faculty of Science, South Valley University, Kena, Egypt \\ e-mail: g_hoshoudy@yahoo.com
}

\begin{abstract}
Results of investigation on the transport of vorticity in Rivlin-Ericksen viscoelastic fluid in the presence of suspended magnetic particles is presented here. Equations governing the transport of vorticity in Rivlin-Ericksen viscoelastic fluid in the presence of suspended magnetic particles are obtained from the equations of magnetic fluid flow. From these equations it follows that the transport of solid vorticity is coupled with the transport of fluid vorticity. Further, we find that because of thermokinetic process, fluid vorticity may exist in the absence of solid vorticity, but when fluid vorticity is zero, then solid vorticity is necessarily zero. A two-dimensional case is also studied and found that the fluid vorticity is indirectly influenced by the temperature and the magnetic field gradient.
\end{abstract}

Keywords: Rivlin-Ericksen viscoelastic fluid, suspended magnetic particles, vorticity

\section{Introduction}

A magneto-rheological fluid contains particles of magnetic materials mixed in a liquid that acts as a carrier. Under normal conditions, the material behaves like a viscous fluid. When it is exposed to a magnetic field, the particles inside align and it responds to the field, exhibiting magnetized behaviour. There are a number of uses for magnetic fluids, ranging from medicine to industrial manufacturing. It is, therefore, a two-phase system consisting of solid and liquid phases. We assume that the liquid phase is non-magnetic in nature and magnetic force acts only on the magnetic particles. Thus, the magnetic force changes the velocity of the magnetic particles. Consequently, the dragging force acting on the carrier liquid is changed and thus the flow of carrier liquid is also influenced by the magnetic force. Because of the relative velocity between the solid and liquid particles, the net effect of the particles suspended in the fluid is extra dragging force acting on the system. Taking this force into consideration, Saffman (1962) proposed the equations of the flow of suspension of non-magnetic particles. These equations were subsequently modified to describe the flow of magnetic fluid with the magnetic body force $\mu_{0} M \nabla H$ taken into account by Wagh (1991). Wagh and Jawandhia (1996) have studied the transport of vorticity in a magnetic fluid. Yan and Koplik (2009) have studied the transport and sedimentation of suspended particles in inertial pressure-driven flow.

In all the above studies, the fluid was considered as Newtonian, but many industrially important fluids (molten plastics, polymers, pulps and foods) exhibit a non-Newtonian fluid behaviour. Many common materials (paints and plastics) and more exotic ones (silicic magma, saturated soils, and the Earth's lithosphere) behave as viscoelastic fluids. With the growing importance of non-Newtonian materials in various manufacturing and processing industries, considerable effort has been directed towards understanding their flow. The stability of a horizontal layer of 
Maxwell's viscoelastic fluid heated from below has been investigated by Vest and Arpaci (1969). The nature of the instability and other factors can affect viscoelastic fluids differently than Newtonian fluids. For example, Bhatia and Steiner (1972) have considered the effect of a uniform rotation on the thermal instability of Maxwellian viscoelastic fluid, where rotation is found to have a destabilizing effect. This is in contrast to the thermal instability of a Newtonian fluid where rotation has a stabilizing effect. The thermal instability of an Oldroydian viscoelastic fluid acted on by a uniform rotation has been studied by Sharma (1976). There are many viscoelastic fluids that cannot be characterized by Maxwell's or Oldroyd's constitutive relations. One such fluid is Rivlin-Ericksen viscoelastic fluid, having relevance and importance in geophysical fluid dynamics, chemical technology and petroleum industry. Rivlin and Ericksen (1955) have studied the stress-deformation relaxation for isotropic materials. Garg et al. (1994) have studied the drag on a sphere oscillating in conducting dusty Rivlin-Ericksen viscielastic fluid. Thermal instability in Rivlin-Ericksen viscoelastic fluid in the presence of rotation and magnetic field, separately, has been investigated by Sharma and Kumar (1996, 1997b). Sharma and Kumar (1997a) have studied the hydromagnetic stability of two Rivlin-Ericksen viscoelastic superposed conducting fluids and the analysis has been carried out, for two highly viscous fluids of equal kinematic viscosities and equal kinematic viscoelasticities wherein it was found that the stability criterion is independent of the effects of viscosity and viscoelasticity and is dependent on the orientation and magnitude of the magnetic field. The stability of two superposed Rivlin-Ericksen viscoelastic fluids in the presence of suspended particles has been considered by Kumar and Singh (2006). Kumar et al. (2007) have studied the hydrodynamic and hydromagnetic stability of two stratified Rivlin-Ericksen viscoelastic superposed fluids.

Keeping in mind the importance of viscoelastic fluids in modern technology and industries, the present paper attempts to study the transport of vorticity in magnetic Rivlin-Ericksen viscoelastic fluid-particle mixtures by using the equations proposed by Wagh and Jawandhia (1996).

\section{Basic assumptions and magnetic body force}

The particles of magnetic material are much larger than the molecules of the carrier liquid. Accordingly, we consider the limit of a microscopic volume element in which the fluid can be assumed to be a continuous medium and the magnetic particles must be treated as discrete entities. If we consider a cell of magnetic fluid containing a larger number of magnetic particles, then we must consider the microrotation of the cell in addition to its translations as a point mass. We must, therefore, assign average velocity $\mathbf{q}_{d}$ and the average angular velocity $\boldsymbol{\omega}$ to the cell. But, here as an approximation, we neglect the effect of microrotation. We also assume the following:

(i) The free current density $\mathbf{J}$ is negligible, and $\mathbf{J} \times \mathbf{B}$ is insignificant.

(ii) The magnetic field is curl free i.e. $\nabla \times \mathbf{H}=\mathbf{0}$.

(iii) The liquid compressibility is unimportant in many practical situations. Hence, the contribution due to magnetic friction can be neglected. The remaining force of the magnetic field is referred as magnetization force.

(iv) All time-dependent magnetization effects in the fluid (such as hysteresis) are negligible, and the magnetization $\mathbf{M}$ is collinear with $\mathbf{H}$.

From electromagnetic theory, the force per unit volume (in MKS units) on a piece of magnetized material of magnetization $\mathbf{M}$ (i.e. dipole moment per unit volume) in the field of magnetic intensity $\mathbf{H}$ is $\mu_{0}(\mathbf{M} \cdot \nabla) \mathbf{H}$, where $\mu_{0}$ is the free space permeability. 
Here we note that

$$
\nabla \cdot \mathbf{a}=\frac{\partial a_{1}}{\partial x}+\frac{\partial a_{2}}{\partial y}+\frac{\partial a_{3}}{\partial z} \quad \mathbf{a} \cdot \nabla=a_{1} \frac{\partial}{\partial x}+a_{2} \frac{\partial}{\partial y}+a_{3} \frac{\partial}{\partial z}
$$

where $\mathbf{a}=a_{1} \mathbf{i}+a_{2} \mathbf{j}+a_{3} \mathbf{k}$.

Using assumption (iv), we obtain

$$
\mu_{0}(\mathbf{M} \cdot \nabla) \mathbf{H}=\frac{\mu_{0} M}{H}(\mathbf{H} \cdot \nabla) \mathbf{H}
$$

where $M=|\mathbf{M}|$ and $H=|\mathbf{H}|$.

But by assumption (ii), we have

$$
(\mathbf{H} \cdot \nabla) \mathbf{H}=\frac{1}{2} \nabla(\mathbf{H} \cdot \mathbf{H})-\mathbf{H} \times(\nabla \times \mathbf{H})=\frac{1}{2} \nabla(\mathbf{H} \cdot \mathbf{H})
$$

Hence

$$
\mu_{0}(\mathbf{M} \cdot \nabla) \mathbf{H}=\left(\frac{\mu_{0} M}{H}\right) \frac{1}{2} \nabla(\mathbf{H} \cdot \mathbf{H})=\mu_{0} M \nabla H
$$

The magnetic body force therefore becomes (Rosensweig, 1997)

$$
\mathbf{f}_{m}=\mu_{0} M \nabla H
$$

\section{Derivation of the equations governing the vorticity transport in a magnetic Rivlin-Ericksen viscoelastic fluid}

Let $\Gamma_{i j}, \tau_{i j}, e_{i j}, \delta_{i j}, u_{i}, x_{i}, p, \mu$ and $\mu^{\prime}$ denote the stress tensor, shear stress tensor, rate-ofstrain tensor, Kronecker delta, velocity vector, position vector, isotropic pressure, viscosity and viscoelasticity, respectively. The constitutive relations for the Rivlin-Ericksen viscoelastic fluid (Rivlin and Ericksen, 1955; Sharma and Kumar, 1997a) are

$$
\Gamma_{i j}=-p \delta_{i j}+\tau_{i j} \quad \tau_{i j}=2\left(\mu+\mu^{\prime} \frac{\partial}{\partial t}\right) e_{i j} \quad e_{i j}=\frac{1}{2}\left(\frac{\partial u_{i}}{\partial x_{j}}+\frac{\partial u_{j}}{\partial x_{i}}\right)
$$

To describe the flow of a magnetic fluid by including the body force $\mu_{0} M \nabla H$ acting on the suspended magnetic particles, Wagh (1991) modified the Saffman's equations for flow of suspension. The equations for the flow of suspended magnetic particles and the flow of Rivlin-Ericksen viscoelastic fluid in which magnetic particles are suspended are therefore written as

$$
\begin{aligned}
& m N\left(\frac{\partial \mathbf{V}}{\partial t}+(\mathbf{V} \cdot \nabla) \mathbf{V}\right)=m N \mathbf{g}+\mu_{0} M \nabla H+K N(\mathbf{u}-\mathbf{V}) \\
& \rho\left(\frac{\partial \mathbf{u}}{\partial t}+(\mathbf{u} \cdot \nabla) \mathbf{u}\right)=-\nabla P+\rho \mathbf{g}+\left(\mu+\mu^{\prime} \frac{\partial}{\partial t}\right) \nabla^{2} \mathbf{u}+K N(\mathbf{V}-\mathbf{u})
\end{aligned}
$$

where $P, \rho, \mathbf{u}\left(u_{x}, u_{y}, u_{z}\right), \mathbf{g}(0,0,-g), \mathbf{V}(l, r, s), m$ and $N(\bar{x}, t)$ respectively denote the pressure minus hydrostatic pressure, density, velocity of fluid particles, gravity force, velocity of solid particles, particle mass and particle number density. Moreover, $\bar{x}=(x, y, z)$, and $K=6 \pi \mu \eta$, where $\eta$ is the particle radius, is the Stokes' drag coefficient.

If we assume that the particle has a uniform spherical shape and that the velocity relative to the fluid is small, then in the equations of motion for the viscoelastic fluid, because of the presence of suspended particles, an additional force term appears proportional to the velocity difference between the suspended particles and the fluid. Since the force exerted by the fluid 
on the suspended particles is equal and opposite to that exerted by the particles on the fluid, an additional force term equal in magnitude but opposite in sign appears in the equations of motion for the suspended particles. We neglect the buoyancy force on the particles. This force is proportional to the quotient of $\rho$ and the particle density, and an analysis in the case of free-free boundary conditions (no tangential stresses) shows that its small stabilizing effect is negligible. We also assume that the distances between particles are quite large compared with their diameter, and we therefore also ignore particle interactions.

By making use of the Lagrange's vector identities

$$
\left(\mathbf{q}_{d} \cdot \nabla\right) \mathbf{q}_{d}=\frac{1}{2} \nabla q_{d}^{2}-\mathbf{q}_{d} \times \boldsymbol{\Omega} \quad(\mathbf{q} \cdot \nabla) \mathbf{q}=\frac{1}{2} \nabla q^{2}-\mathbf{q} \times \boldsymbol{\Omega}_{1}
$$

Equations (3.1) become

$$
\begin{aligned}
& m N\left[\frac{\partial \mathbf{V}}{\partial t}-(\mathbf{V} \times \boldsymbol{\Omega})\right]=-\nabla m N g z-\frac{1}{2} m N \nabla \mathbf{V}^{2}+\mu_{0} M \nabla H+K N(\mathbf{u}-\mathbf{V}) \\
& \rho\left[\frac{\partial \mathbf{u}}{\partial t}-\left(\mathbf{u} \times \boldsymbol{\Omega}_{1}\right)\right]=-\nabla P-\nabla \rho g z-\frac{1}{2} \rho \nabla \mathbf{u}^{2}+\left(\mu+\mu^{\prime} \frac{\partial}{\partial t}\right) \nabla^{2} \mathbf{u}+K N(\mathbf{V}-\mathbf{u})
\end{aligned}
$$

where $\boldsymbol{\Omega}=\nabla \times \mathbf{V}$ and $\boldsymbol{\Omega}_{1}=\nabla \times \mathbf{u}$ are solid vorticity and fluid vorticity.

Taking the curl of these equations and recalling that the curl of a gradient is identically equal to zero, we obtain

$$
\begin{aligned}
& m N\left[\frac{\partial \boldsymbol{\Omega}}{\partial t}-(\nabla \times \mathbf{V} \times \boldsymbol{\Omega})\right]=\mu_{0} \nabla \times M \nabla H+K N\left(\boldsymbol{\Omega}_{1}-\boldsymbol{\Omega}\right) \\
& \rho\left[\frac{\partial \boldsymbol{\Omega}_{1}}{\partial t}-\left(\nabla \times \mathbf{u} \times \boldsymbol{\Omega}_{1}\right)\right]=\left(\mu+\mu^{\prime} \frac{\partial}{\partial t}\right) \nabla^{2} \boldsymbol{\Omega}_{1}+K N\left(\boldsymbol{\Omega}-\boldsymbol{\Omega}_{1}\right)
\end{aligned}
$$

By making use of the vector identities

$$
\begin{aligned}
& \nabla \times(\mathbf{V} \times \boldsymbol{\Omega})=(\boldsymbol{\Omega} \cdot \nabla) \mathbf{V}-(\mathbf{V} \cdot \nabla) \boldsymbol{\Omega}+\mathbf{V} \nabla \cdot \boldsymbol{\Omega}-\boldsymbol{\Omega} \nabla \cdot \mathbf{V}=(\boldsymbol{\Omega} \cdot \nabla) \mathbf{V}-(\mathbf{V} \cdot \nabla) \boldsymbol{\Omega} \\
& \nabla \times\left(\mathbf{u} \times \boldsymbol{\Omega}_{1}\right)=\left(\boldsymbol{\Omega}_{1} \cdot \nabla\right) \mathbf{u}-(\mathbf{u} \cdot \nabla) \boldsymbol{\Omega}_{1}+\mathbf{u} \nabla \cdot \boldsymbol{\Omega}_{1}-\boldsymbol{\Omega}_{1} \nabla \cdot \mathbf{u}=\left(\boldsymbol{\Omega}_{1} \cdot \nabla\right) \mathbf{u}-(\mathbf{u} \cdot \nabla) \boldsymbol{\Omega}_{1}
\end{aligned}
$$

Equations (3.4) become

$$
\begin{aligned}
& m N \frac{D \boldsymbol{\Omega}}{D t}=\mu_{0} \nabla \times M \nabla H+m N(\boldsymbol{\Omega} \cdot \nabla) \mathbf{V}+K N\left(\boldsymbol{\Omega}_{1}-\boldsymbol{\Omega}\right) \\
& \frac{D \boldsymbol{\Omega}_{1}}{D t}=\left(\nu+\nu^{\prime} \frac{\partial}{\partial t}\right) \nabla^{2} \boldsymbol{\Omega}_{1}+\left(\boldsymbol{\Omega}_{1} \cdot \nabla\right) \mathbf{u}+\frac{K N}{\rho}\left(\boldsymbol{\Omega}-\boldsymbol{\Omega}_{1}\right)
\end{aligned}
$$

where $\nu$ and $\nu^{\prime}$ are kinematic viscosity and kinematic viscoelasticity, respectively and $\frac{D}{D t} \equiv \frac{\partial}{\partial t}+(\mathbf{V} \cdot \nabla)$ is the convective derivative.

In equation $(3.6)_{1}$

$$
\nabla \times(M \nabla H)=(\nabla M \times \nabla H)+(M \nabla \times \nabla H)
$$

Since the curl of the gradient is zero, the last term in equation (3.7) is zero. Also since $M=M(H, T)$.

Therefore

$$
\nabla M=\left(\frac{\partial M}{\partial H}\right) \nabla H+\left(\frac{\partial M}{\partial T}\right) \nabla T
$$

By making use of (3.8), equation (3.7) becomes

$$
\nabla \times(M \nabla H)=\left(\frac{\partial M}{\partial H}\right) \nabla H \times \nabla H+\left(\frac{\partial M}{\partial T}\right) \nabla T \times \nabla H
$$


The first term on the right hand side of this equation is clearly zero, hence we get

$$
\nabla \times(M \nabla H)=\left(\frac{\partial M}{\partial T}\right) \nabla T \times \nabla H
$$

Substituting this expression in equation $(3.6)_{1}$, we obtain

$$
m N \frac{D \boldsymbol{\Omega}}{D t}=\mu_{0}\left(\frac{\partial M}{\partial T}\right) \nabla T \times \nabla H+m N(\boldsymbol{\Omega} \cdot \nabla) \mathbf{V}+K N\left(\boldsymbol{\Omega}_{1}-\boldsymbol{\Omega}\right)
$$

Here (3.6) $)_{2}$ and (3.11) are the equations governing the transport of vorticity in magnetic RivlinEricksen viscoelastic fluid-particle mixtures.

In equation (3.11), the first term in the right-hand side i.e. $\mu_{0}(\partial M / \partial T) \nabla T \times \nabla H$ describes the production of vorticity due to thermo-kinetic processes. The last term $K N\left(\boldsymbol{\Omega}_{1}-\boldsymbol{\Omega}\right)$ gives the change in solid vorticity on account of the exchange of vorticity between the liquid and solid.

It follows from equations (3.6) $)_{2}$ and (3.11) that the transport of solid vorticity $\boldsymbol{\Omega}$ is coupled with the transport of fluid vorticity $\boldsymbol{\Omega}_{1}$.

From equation (3.11), we see that if solid vorticity $\boldsymbol{\Omega}$ is zero, then the fluid vorticity $\boldsymbol{\Omega}_{1}$ is not-zero and it is given by

$$
\boldsymbol{\Omega}_{1}=-\frac{\mu_{0}}{K N}\left(\frac{\partial M}{\partial T}\right) \nabla T \times \nabla H
$$

This implies that due to thermo-kinetic processes, fluid vorticity can exist in the absence of solid vorticity.

From equation (3.6) $)_{2}$, we find that if $\boldsymbol{\Omega}_{1}$ is zero, then $\boldsymbol{\Omega}$ is also zero. This implies that when fluid vorticity is zero, then solid vorticity is necessarily zero.

In the absence of suspended magnetic particles, $N$ is zero and magnetization $M$ is also zero. Then, equation (3.11) is identically satisfied and equation $(3.6)_{2}$ reduces to

$$
\frac{D \boldsymbol{\Omega}_{1}}{D t}=\left(\nu+\nu^{\prime} \frac{\partial}{\partial t}\right) \nabla^{2} \boldsymbol{\Omega}_{1}+\left(\boldsymbol{\Omega}_{1} \cdot \nabla\right) \mathbf{q}
$$

This equation is the vorticity transport equation. The last term on the right hand side of equation (3.13) represents the rate at which $\boldsymbol{\Omega}_{1}$ varies for a given particle, when the vortex lines move with the fluid, the strengths of the vortices remaining constant. The first term represents the rate of dissipation of vorticity through friction (resistance) and rate of change of vorticity due to fluid viscoelasticity.

\subsection{Two-dimensional case}

Here we consider the two-dimensional case:

Let

$$
\mathbf{V}=v_{x}(x, y) \mathbf{i}+v_{y}(x, y) \mathbf{j} \quad \mathbf{u}=u_{x}(x, y) \mathbf{i}+u_{y}(x, y) \mathbf{j}
$$

where components $v_{x}, v_{y}$ and $u_{x}, u_{y}$ are functions of $x, y$ and $t$, then

$$
\boldsymbol{\Omega}=\Omega_{z} \mathbf{k} \quad \Omega_{1}=\Omega_{1 z} \mathbf{k}
$$

In two-dimensional case, equation (3.11) becomes

$$
\frac{D \Omega_{z}}{D t}=\frac{\mu_{0}}{m N}\left(\frac{\partial M}{\partial T}\right)\left(\frac{\partial T}{\partial x} \frac{\partial H}{\partial y}-\frac{\partial H}{\partial x} \frac{\partial T}{\partial y}\right)+\frac{K}{m}\left(\Omega_{1 z}-\Omega_{z}\right)
$$


and equation $(3.6)_{2}$ similarly becomes

$$
\frac{D \Omega_{1 z}}{D t}=\nu \nabla^{2}\left(\Omega_{1 z}\right)+\nu^{\prime} \frac{\partial}{\partial t} \nabla^{2}\left(\Omega_{1 z}\right)+\frac{K N}{\rho}\left(\Omega_{z}-\Omega_{1 z}\right)
$$

since it can be easily verified that

$$
(\boldsymbol{\Omega} \cdot \nabla) \mathbf{V}=0 \quad\left(\boldsymbol{\Omega}_{1} \cdot \nabla\right) \mathbf{u}=0
$$

The first term on the right hand side of equation (3.17) is the change of fluid vorticity due to internal friction (resistance). The second term is the rate of change of fluid vorticity due to fluid viscoelasticity and the third term is change in fluid vorticity due to the exchange of vorticity between solid and liquid. Equation (3.17) does not explicitly involve the term representing change of vorticity due to magnetic field gradient and/or temperature gradient. But equation (3.16) shows that solid vorticity $\Omega_{z}$ depends on these factors. Hence, it follows that the fluid vorticity is indirectly influenced by the temperature and the magnetic field gradient.

In the absence of magnetic particles, $N$ is zero and magnetization $M$ is also zero. Equation (3.16) is therefore satisfied identically, and equation (3.17) reduces to the classical equation for the transport of fluid vorticity. If we consider a suspension of non-magnetic particles instead of a magnetic fluid, then the corresponding equation for the transport of vorticity may be obtained by setting $M$ equal to zero in the equations governing the transport of vorticity in magnetic fluids. If magnetization $M$ of the magnetic particles is independent of temperature, then the first term of equations (3.11) and (3.16) vanishes, and the equations governing the transport of vorticity in a magnetic fluid become the same as those governing the transport of vorticity in non-magnetic suspensions.

If the temperature gradient $\nabla T$ vanishes or if the magnetic field gradient $\nabla H$ vanishes or if $\nabla T$ is parallel to $\nabla H$, then also the first term in (3.11) and (3.16) vanishes. We thus see that the transport of vorticity in a magnetic fluid is also the same as the transport of vorticity in non-magnetic suspension.

\section{Acknowledgements}

The authors are grateful to the learned referee for his useful technical comments and valuable suggestions, which led to a significant improvement of the paper.

\section{References}

1. Bhatia P.K., Steiner J.M., 1972, Convective instability in a rotating viscoelastic fluid layer, Zeitschrift für Angewandte Mathematik und Mechanik, 52, 321-324

2. Garg A., Srivastava R.K., Singh K.K., 1994, Drag on a sphere oscillating in conducting dusty Rivlin-Ericksen elastico-viscous liquid, Proceedings of the National Academy of Sciences, India, 64A, 355-361

3. Kumar P., Lal R., Singh M., 2007, Hydrodynamic and hydromagnetic stability of two stratified Rivlin-Ericksen elastico-viscous superposed fluids, International Journal of Applied Mechanics and Engineering, 12, 645-653

4. Kumar P., Singh G.J., 2006, Stability of two superposed Rivlin-Ericksen viscoelastic fluids in the presence of suspended particles, Romanian Journal of Physics, 51, 927-935

5. Rivlin R.S., ERICKSEn J.L., 1955, Stress-deformation relaxations for isotropic materials, Archive for Rational Mechanics and Analysis, 4, 323-425

6. Rosensweig R.E., 1997, Ferrohydrodynamics, Dover Publications, Inc. Mineola, New York

7. Saffman P., 1962, On the stability of a laminar flow of a dusty gas, Journal of Fluid Mechanics, 13, $120-128$ 
8. Sharma R.C., 1976, Effect of rotation on thermal instability of a viscoelastic fluid, Acta Physica Hungarica, 40, 11-17

9. Sharma R.C., Kumar P., 1996, Effect of rotation on thermal instability in Rivlin-Ericksen elastico-viscous fluid, Zeitschrift für Naturforschung, 51a, 821-824

10. Sharma R.C., Kumar P., 1997a, Hydromagnetic stability of Rivlin-Ericksen elastico-viscous superposed conducting fluids, Zeitschrift für Naturforschung, 52a, 528-532

11. Sharma R.C., Kumar P., 1997b, Thermal instability in Rivlin-Ericksen elastico-viscous fluid in hydromagnetics, Zeitschrift für Naturforschung, 52a, 369-371

12. Vest C.M., Arpaci V.S., 1969, Overstability of a viscoelastic fluid layer heated from below, Journal of Fluid Mechanics, 36, 613-619

13. WAGH D.K., 1991, A mathematical model of magnetic fluid considered as two-phase system, Proceedings of International Symposium on Magnetic Fluids, R.E.C. Kurukshetra (Edit.), India, Sept. 21-23, 182

14. Wagh D.K., JaWAndhia A., 1996, Transport of vorticity in magnetic fluid, Indian Journal of Pure and Applied Physics, 34, 338-340

15. YAN Y., Koplik J., 2009, Transport and sedimentation of suspended particles in inertial pressuredriven flow, Physics of Fluids, 21, 013301

Manuscript received December 24, 2014; accepted for print July 13, 2015 